Documentation et bibliothèques

DOCUMENTATION BIBLIOTHËQUES

\title{
Services d'information : bouleversements, enjeux et stratégies
}

\section{Joanne Cournoyer}

Volume 42, numéro 3, juillet-septembre 1996

URI : https://id.erudit.org/iderudit/1033260ar

DOI : https://doi.org/10.7202/1033260ar

Aller au sommaire du numéro

Éditeur(s)

Association pour l'avancement des sciences et des techniques de la documentation (ASTED)

ISSN

0315-2340 (imprimé)

2291-8949 (numérique)

Découvrir la revue

Citer ce document

Cournoyer, J. (1996). Services d'information : bouleversements, enjeux et stratégies. Documentation et bibliothèques, 42(3), 107-108.

https://doi.org/10.7202/1033260ar

Tous droits réservés (C) Association pour l'avancement des sciences et des techniques de la documentation (ASTED), 1996
Ce document est protégé par la loi sur le droit d'auteur. L'utilisation des services d'Érudit (y compris la reproduction) est assujettie à sa politique d'utilisation que vous pouvez consulter en ligne.

https://apropos.erudit.org/fr/usagers/politique-dutilisation/ 


\title{
Services d'information : bouleversements, enjeux et stratégies.
}

\author{
Joanne Cournoyer * \\ Présidente de l'ASTED
}

Lors du dernier congrès en octobre dernier, nous avons analysé les diverses frontières technologiques, politiques, professionnelles, humaines et économiques et nous avons exploré les divers moyens de les franchir. Cette année, nous poursuivrons la réflexion en examinant les changements qui modifient les environnements des services d'information, bouleversent à la fois les individus et les organisations et exigent de nouvelles stratégies qui font appel à la créativité, à la collaboration et forcent à mesurer les objectifs réalisés et le succès obtenu.

Depuis quelques années, la principale caractéristique de notre société et de notre vie professionnelle est le changement. Nous sommes en pleine révolution depuis que le développement et la diffusion de l'information sont devenus les activités principales de productivité et de pouvoir de notre société. La révolution amène forcément la rupture avec certaines approches traditionnelles qui ne correspondent pas à cette nouvelle ère ou à cette «troisième vague».

Les changements technologiques bouleversent notre vie : magasinage en ligne, Internet, Intranet, messages de publicité ciblés ou le "prêt-à-surfer» (Cloutier, Jean-Pierre. 1996. Le prêt-àsurfer). Dans nos organisations, les outils, l'environnement, le rôle du spécialiste en information, absolument tout est en bouleversement. On doit sans cesse mettre l'équipement à jour, les ressources humaines doivent recevoir la formation nécessaire pour faire face aux divers changements et les apprivoiser, le service doit continuer à être de qualité et malgré les embûches et les restrictions, on souhaite continuer à remplir sa mission.
Nos services d'information vivent et respirent au rythme des nouvelles technologies de l'information. L'an dernier, Laurent Laplante nous invitait à ne pas oublier l'espace humain de notre profession et rappelait que la population a besoin de nous pour rattraper le courant. Un sondage réalisé en juin dernier auprès de 1069 personnes révèle que $« 41 \%$ de la population québécoise n'a jamais entendu parler d'Internet, $11 \%$ des répondants francophones croient qu'Internet est une mode passagère alors que seulement $4 \%$ des non-francophones le croient» (Gagnon, Gilles. 1996. Les affaires). Ce résultat est sans aucun doute lié à la langue du contenu d'Internet. Le développement de l'autoroute se doit de mettre l'accent sur l'individu et les communautés. On doit pouvoiry retrouvernos valeurs et notre culture. «ll faut que le contenu ait quelque chose d'attrayant pour qu'il retienne l'attention" dit Adel Le Zaïm du CRIM et Jean-Claude Guédon souligne que «nous sommes actuellement dans une période d'appropriation et d'apprentissage.»

Le sondage fait ressortir clairement que plus les répondants ont un revenu familial élevé, plus ils ont entendu parler d'Internet. «Les gens qui ont des revenus élevés ont en général un accès plus grand à l'information et elles se tiennentà l'avantgarde des nouvelles technologies». II y a donc ceux qui savent et ceux qui n'ont pas les moyens de savoir. Les frontières sont toujours là pour l'individu. Avons-nous un rôle à jouer dans cet accès au savoir? Pouvons-nous assurer cet accès dans un contexte de restrictions?

Nous faisons face à plusieurs défis. Comment se positionner dans cette nou- velle industrie? Comment faire reconnaître la valeur de nos services à l'intérieur de nos organisations? Comment assurer l'accès au citoyen, notre usager? Comment s'approprier ces nouvelles technologies? Comment s'assurer que les individus et les sociétés y trouvent leur compte? Comment redéfinir notre rôle devant ces technologies qui sont là pour rester? Avons-nous toujours la même mission?

En tant que spécialiste de l'information, nous devons nous interroger aussi sur ce que nous sommes, sur notre rôle et les compétences à développer afin de bien choisir nos approches et nos façons de faire. En tant qu'organisation à l'ère des changements, nous devons porter un regard sur ce que nous faisons, analyser la façon dont nous le faisons, trouver quels sont nos facteurs clés de succès et trouver les moyens de se repositionner afin de rester parmi les meilleurs joueurs et les décideurs.

Une organisation peut mesurer et gérer son succès en s'interrogeant sur sa vision ou ses facteurs clés de succès, ses cibles ou ses objectifs et les indicateurs de performance. Longtemps on a compilé des données sans pour autant les utiliser comme outils de mesure reliés à nos objectifs. Enjeux et priorités: nous sommes à l'heure des contraintes et des choix. Dans cette ère de changements et de contraintes budgétaires, il est important qu'un service documentaire mesure son succès dans la réalisation de ses objectifs. II faut faire des choix et se doter d'outils pour prendre des décisions qui assureront la réalisation de la mission.

\footnotetext{
* Joanne Cournoyer est gestionnaire de projets à la Cité collégiale, Ottawa (Ontario).
} 
Le savoir et l'information étant devenus prioritaires dans notre société, les organisations reconnaissent que les ressources humaines deviennent de plus en plus une ressource essentielle de leur organisation. Une théorie à la page dans les entreprises est celle de l'équilibre. Selon cette théorie, les quatre facteurs clés dans une organisation sont : l'excellence des employés, la satisfaction de la clientèle, une bonne gestion financière et l'excellence des processus. Ces quatre facteurs s'influencent mutuellement, guidés par le leadership de la direction. L'équilibre s'installe quand ces quatre éléments ont la même importance au sein d'une organisation ce qui se traduit par des ressources réparties de façon équilibrée entre les éléments. Ainsi, l'équilibre de ces quatre facteurs favorise la réalisation de la mission.
D'autres clés vous seront fournies lors du congrès dont celle de la collaboration. Notre association a adopté cette nouvelle valeur qui se concrétise dans de nombreuses activités: collaboration avec diverses associations dans la réalisation du colloque portant sur la bibliothèque au coeur de la société d'information, partenaire dans le projet ALARM, partenaire dans la défense des droits de l'individu à l'accès aux nouvelles technologies, collaboration avec d'autres associations dans la présentation d'un mémoire sur l'autoroute de l'information, participation au projet Biblionet, etc. Notre association tente de franchir les frontières et de créer des alliances et des partenariats qui assureront la visibilité et la viabilité des services d'information. L'avenir est à la globalisation, sachons nous aussi lire notre environnement et faire des alliances qui nous assureront une présence dans cette société en changement.

Le thème choisi pour le congrès 96 est un thème d'actualité car notre société toute entière est à l'heure des bouleversements, des enjeux et des priorités. Tous, nous nous interrogeons sur les clés qui nous assureront un positionnement stratégique au sein de cette nouvelle société en révolution.

«En préservant la part utile de notre présent, il nous faut préparer l'avenir en intégrant les changements requis» (Curran, William M. 1995. Documentation et biliothèques 41(3): 135-136). Le congrès de 1996 à Montréal nous aidera à faire des pas rapides dans la bonne direction.

\section{PERIODICA}

- PERIODICA Abonnements

10000 titres (magazines, périodiques et journaux du monde entier)

\section{- PERIODICA Vidéo}

1500 titres (arts, sciences, littérature, voyage, jeunesse, cinéma de répertoire)

\section{- BIBLIORAMA}

Tous les livres disponibles de langue française distribués au Canada

\section{- BIBLIODATA}

Banque de données

des Livres disponibles canadiens de langue française 45000 titres, 516 éditeurs, 250 distributeurs

\section{- Partenaire CEDROM-SNI}

600 banques de données sur CD-ROM

(actualité, affaires, sciences et technologies, santé, médecine, éducation)

Accès direct à plus de 1250 sources

d'information électronique

Demandez nos catalogues : La réponse à vos recherches d'outils pédagogiques de langue française.

PERIODICA INC.

Case postale 444, Outremont

Québec, Canada H2V 4R6
Tél. : (514) 274-5468

Fax : (514) 274-0201

Tout le Canada : 1-800-361-1431 